\title{
Reliability and sensitivity to change of the timed standing balance test in children with down syndrome
}

\author{
Vencita Priyanka Aranha, Asir John Samuel, Shikha Saxena ${ }^{1}$ \\ Department of Pediatric Physiotherapy, Maharishi Markandeshwar Institute of Physiotherapy and Rehabilitation, Maharishi Markandeshwar University, \\ Ambala, Haryana, India, ' ${ }^{2}$ epartment of Graduate Studies, School of Physical and Occupational Therapy, McGill University, Montreal, Quebec, Canada
}

\begin{abstract}
Objective: To estimate the reliability and sensitivity to change of the timed standing balance test in children with Down syndrome (DS). Methods: It was a nonblinded, comparison study with a convenience sample of subjects consisting of children with DS $(n=9)$ aged 8-17 years. The main outcome measure was standing balance which was assessed using timed standing balance test, the time required to maintain in four conditions, eyes open static, eyes closed static, eyes open dynamic, and eyes closed dynamic. Results: Relative reliability was excellent for all four conditions with an Interclass Correlation Coefficient (ICC) ranging from 0.91 to 0.93 . The variation between repeated measurements for each condition was minimal with standard error of measurement (SEM) of 0.21-0.59 s, suggestive of excellent absolute reliability. The sensitivity to change as measured by smallest real change (SRC) was $1.27 \mathrm{~s}$ for eyes open static, $1.63 \mathrm{~s}$ for eyes closed static, 0.58 s for eyes open dynamic, and $0.61 \mathrm{~s}$ for eyes closed static. Conclusions: Timed standing balance test is an easy to administer test and sensitive to change with strong absolute and relative reliabilities, an important first step in establishing its utility as a clinical balance measure in children with DS.
\end{abstract}

Key words: Absolute reliability, adolescent, children, postural stability psychometric properties, relative reliability

\section{Introduction}

Down syndrome (DS) is one of the most common chromosomal birth defects in children ${ }^{[1]}$ Often postural instability plays a major contributing role to the motor disorders in children with DS. ${ }^{[2]}$ Deficits in postural control in DS are often associated with problems in motor coordination and sensory-motor integration. Other neuro-muscular anomalies, such as hypotonia, retained primary reflexes, and slow performance of volitional reaction not only leads to delayed development in motor and cognitive functions, but also problems with body balance. ${ }^{[2]}$ Postural stability is a prerequisite for

\section{Address for correspondence:}

Dr. Asir John Samuel, Maharishi Markandeshwar Institute of Physiotherapy and Rehabilitation, Maharishi Markandeshwar University, Mullana - 133 207, Haryana, India.

E-mail: asirjohnsamuel@mmumullana.org

\begin{tabular}{|l|l|}
\hline \multicolumn{2}{|c|}{ Access this article online } \\
\hline Quick Response Code: & Website: \\
\hline & www.ruralneuropractice.com \\
\cline { 1 - 2 } & \\
\hline & \\
\hline
\end{tabular}

performing a number of complex gross motor activities and hence affects the child's interaction with peers, play activities, and participation in social activities. To address these problems, a lot of emphasis is now being given to community-based balance and strength training programs in individuals with DS that encourages participation in leisure activities and interaction with peers..$^{[3-6]}$ Therefore, accurate measures of static and dynamic balance in a community or school based setting are required to assess the effectiveness of the balance training and to examine the severity of balance problems. ${ }^{[7]}$

Villamonte et al. estimated the reliability of 16 static and dynamic balance tests in individuals with DS aged 5-31 years and found that only 3 tests were reliable in young males and females. ${ }^{[8]}$ However, the results of this study cannot be generalized to the population with

This is an open access article distributed under the terms of the Creative Commons Attribution-NonCommercial-ShareAlike 3.0 License, which allows others to remix, tweak, and build upon the work non-commercially, as long as the author is credited and the new creations are licensed under the identical terms.

For reprints contact: reprints@medknow.com

How to cite this article: Aranha VP, Samuel AJ, Saxena S. Reliability and sensitivity to change of the timed standing balance test in children with down syndrome. J Neurosci Rural Pract 2016;7:77-82. 
DS because of three reasons. First, this study included a wide age range and insufficient sample size. As children grow into adolescents and adults, there occurs maturation of the organizational processes influencing balance control resulting in large differences in their performances on balance measures. ${ }^{[9,10]}$ Therefore, to obtain better estimates of psychometric properties of a balance measure, it must be evaluated in a specific age group. ${ }^{[8]}$ Second, the reliability of the balance tests was computed only in terms of Interclass Correlation Coefficients (ICC). In recent years, several authors have recommended that no single reliability estimate should be used for reliability studies. ${ }^{[11]}$ For example, ICC or Pearson's correlation coefficient values are affected by sample heterogeneity to such a degree that a high correlation may still mean unacceptable measurement error. ${ }^{[12]}$ In general, correlation coefficients should be assessed with absolute reliability measures like the standard error of measurement (SEM) because a combination of approaches is more likely to give a true picture of reliability. ${ }^{[11]}$ Third, it did not recommend any standardized balance test that could be used in children and adolescents with DS.

To be applicable in clinical and community-based settings, balance tests must be reliable and valid, use readily available equipment, require a little experience to master, easy to administer and cost effective. Another important consideration is that the balance tests should account for the complexity and multidimensional nature of balance that is determined by three components: (1) The musculoskeletal system (biomechanical components), (2) automatic postural reactions (motor coordination components), and (3) postural orientation (sensory organization components). ${ }^{[13]}$ Therefore, balance assessments should incorporate protocols that can assess these components separately. One of the clinical balance measures that is easy to use and evaluates different balance components is the static and dynamic timed standing balance test (SDSBT). It is a clinical test that has been proven to be appropriate and reliable in healthy adolescents. ${ }^{[14]}$ SDSBT is simple in instrumentation and uses no sophisticated technique to evaluate patients' balance under eyes open and eyes closed standing conditions. ${ }^{[14]}$ It has been tested in different patient populations, but its psychometric properties in measuring balance among the children and adolescents with DS are not yet established. ${ }^{[15,16]}$ To test a clinical measure for its psychometric properties, it is suggested that reliability should be tested for first in a new measurement tool, since the measure will never be valid if it is not adequately consistent in whatever value it indicates from repeated measurements. ${ }^{[12]}$ The purpose of this study was to estimate the sensitivity to change and reliability of SDSBT to assess standing balance in children with DS aged 8-17 years. The sensitivity to change was evaluated to judge whether the measurement error would be small enough to justify the use of SDSBT to detect change over time in children with DS.

\section{Methods}

This was a nonblinded, comparison study conducted on children with DS. The study was approved from the University Review Board on Ethics for research, and the study protocol has been registered in Trials Registry of India (CTRI/2014/08/004932). The study was conducted at a special school for children with disabilities. A prior informed consent was obtained from the school authorities, as well as from all the participants' parents/guardians. Nature and purpose, procedures, risks, and benefits of the study was explained to them while taking due care to avoid any bias. They were ensured that confidentiality of the data would be maintained. The study was conducted according to guidelines for biomedical research on human participants, laid by World Medical Association Declaration of Helsinki at $64^{\text {th }}$ General Assembly meeting, Brazil, October 2013.

\section{Sample characteristics}

The study was designed to recruit nine subjects to have $80 \%$ power with an expected reliability $\left(\rho_{0}\right)$ of $>0.6$ and an expected width of 0.2 at $P=0.05$ level. ${ }^{[17,18]}$ Convenience sampling technique was used to recruit nine children and adolescents with DS (6 boys, 3 girls) aged 8-17 years from a sample of 19 children between September 2014 and November 2014 with pilot trial being conducted in the month of June to August, 2013. Children with DS were included if they could understand and follow a simple command, and stand independently without assistive devices. Subjects were excluded if they reported a history of any musculoskeletal injury 6 weeks prior to recruitment. Every child was shown how the test had to be performed, and a $15 \mathrm{~s}$ practice trial was given in each condition. A digital stopwatch was used to measure the time required to maintain each test position that was recorded to the nearest $1 / 100$ of a second. The test was conducted barefoot to maintain uniformity. All the anthropometric measures (height, weight, waist-hip ratio) were recorded in the beginning of the study.

\section{Procedure}

The static balance was tested by standing on a floor and dynamic balance by standing on a foam $\operatorname{pad}(50 \mathrm{~cm} \times 48 \mathrm{~cm} \times 8 \mathrm{~cm}, 0.8 \mathrm{~kg})$. The test was conducted with four different protocols (eyes open static, eyes closed static, eyes open dynamic, and eyes closed 
dynamic) and the order of each protocol was randomized by lottery method to control measurement bias. A rest for $30 \mathrm{~s}$ was provided between protocols. The dominant leg was identified by asking the subject to kick a ball prior to testing. For all four balance tests, each subject completed three trials on each leg.

\section{Static balance}

Participants were asked to place their hands on their hip and to focus on a target which was placed at the eye level, at a distance of $4 \mathrm{~m}$ from them. They were then asked to stand on their right foot and raise the opposite foot from the floor $\left(90^{\circ}\right.$ knee flexion) as in Figure 1 . The electronic stopwatch was started as soon as the subject raised the foot from the floor and was stopped on the loss of balance. Loss of balance included removal of one hand from the hip, touching the floor with the nonweight-bearing foot, or movement of the weight-bearing foot from its original position on the floor. The same procedure was repeated with closed eyes. The stop watch was stopped on opening of eye and loss of balance.

\section{Dynamic balance}

The same procedure was again repeated on a foam surface [Figure 2] with an eye open and eye closed. Here, the stopwatch was started as above but was stopped on the loss of balance or on the movement of the foam from its original position. Due to small sample size, the assessment was made on three occasions, by the same examiner with 2 days interval between each session.

\section{Data analysis}

The main clinical outcome measure was the time (seconds) required to maintain each position under static and dynamic balance testing conditions. Data analysis was performed using the Statistical Package for Social Science, IBM ${ }^{\circledR}$ SPSS $^{\circledR}$ v20 (IBM

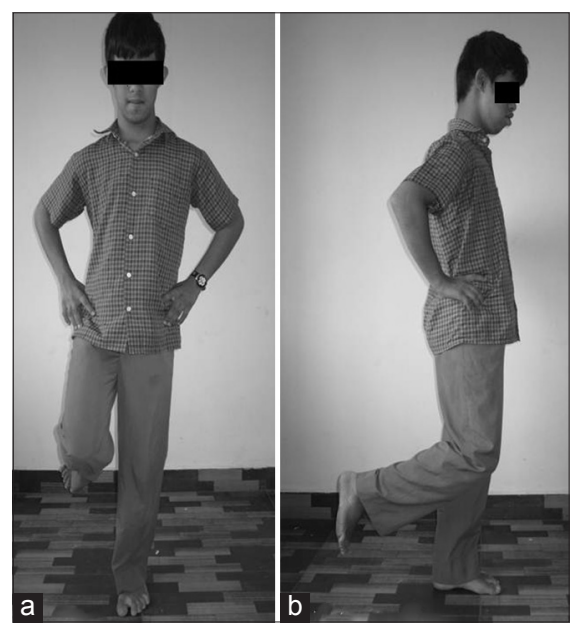

Figure 1: A child with down syndrome performing the timed static standing balance test by standing over the flat surface (a) AP view (b) Lateral view
USA). Normality of collected data was checked using Shapiro-Wilk test. As the data did not follow a normal distribution, the test score data were transformed logarithmically and then back transformed to report the geometric mean and 95\% confidence interval (CI). When data are skewed, geometric mean and $95 \% \mathrm{CI}$ are then appropriate parameters to express the arithmetic mean and standard deviation (SD) $\cdot{ }^{[19]}$ The reliability of the assessment instrument was calculated by estimating its relative and absolute reliability. Relative reliability is the degree to which individuals maintain their position in a sample over repeated measurements and was assessed using $\operatorname{ICC}_{1, \mathrm{k}}(\mathrm{k}=3){ }^{[11]}$ ICC values from 0.75 to 0.89 represented good reliability and $0.90-0.99$ represented excellent reliability. ${ }^{[2]}$ Absolute reliability is the degree to which repeated measurements vary for individuals. It will be assessed using SEM, which is the SD of measurement errors. ${ }^{[11]}$ SEM was used as a measure of absolute reliability and calculated as $\mathrm{SEM}=\mathrm{SD} \times \sqrt{ }\left(\right.$ 1-reliability). ${ }^{[21]}$ Sensitivity to change was calculated using the smallest real change (SRC). Based on the SEM, the SRC with 95\% confidence is calculated as $1.96 \times \sqrt{2} \times$ SEM at $P<0.05 .^{[12]}$ Differences between two consecutive measurements that are greater than the SRC can be interpreted as real change with $95 \%$ certainty. SRC is not the "clinically relevant change," but a clinimetric property of a measurement instrument. ${ }^{[22,23]}$

\section{Results}

A total of nine children with DS were recruited for the study, six boys and three girls. Subject demographics are presented in Table 1. There was no significant difference in any demographic variable between boys and girls. Table 2 shows the geometric mean with $95 \%$ CI, median with range, ICC, SEM, and 95\% SEM of

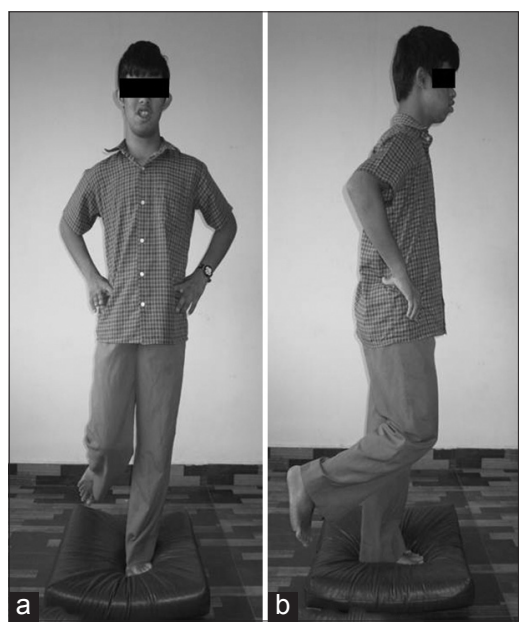

Figure 2: A child with down syndrome performing the timed dynamic standing balance test by standing over the foam surface (a) AP view (b) Lateral view 
timed standing balance test in all four conditions eyes open static, eyes closed static, eyes open dynamic, and eyes closed dynamic. Eyes open static condition had the highest test scores and greatest dispersion of its middle $50 \%$ median values.

All conditions showed excellent ICC values ranging between 0.91 and 0.93. SEM scores and 95\% SEM for eyes open and closed under dynamic conditions were smaller than eyes open and closed under static condition, an important consideration during the comparison of observed scores and true scores for independent condition. For example, the SEM for eyes open dynamic is 0.21 and $95 \%$ SEM is \pm 0.41 . This implies that when a child with DS has scored $4 \mathrm{~s}$ on the eyes open dynamic condition, one can be $95 \%$ sure that his/her true score ranges from 3.59 to 4.41 . Overall, all test conditions demonstrated excellent SEM percentages. Table 2 also shows the SRCs for all test scores. According to the SRC values, a change of at least $2 \mathrm{~s}$ for static balance conditions, and a change of at least $1 \mathrm{~s}$ for dynamic balance conditions is needed between two sessions to be $95 \%$ confident that a real change has occurred.

\section{Discussion}

SDSBT is a clinical test that evaluates balance under static and dynamic balance conditions. This study demonstrated the excellent relative and absolute reliability with an ability to detect relatively small differences over time. The study is carried out with an aim to establish the reliability of static and dynamic balance in the children with DS. The foam pad used for

Table 1: Demographic characteristic of the children with down syndrome

\begin{tabular}{lccr}
\hline Characteristics & $\begin{array}{c}\text { Boys }(\boldsymbol{n}=6) \\
\text { median (range) }\end{array}$ & $\begin{array}{c}\text { Girls }(\boldsymbol{n}=3) \\
\text { median (range) }\end{array}$ & $\boldsymbol{P}$ \\
\hline Age (years) & $14(8-17)$ & $13(9-15)$ & 0.36 \\
Height $(\mathrm{cm})$ & $148.3(131-158)$ & $142(137-144)$ & 0.36 \\
Weight $(\mathrm{kg})$ & $44.1(28.9-67.5)$ & $32.5(32.1-36.6)$ & 0.21 \\
${ }^{*} \mathrm{BMI}$ & $19.9(16.5-28.1)$ & $17.1(15.7-18.2)$ & 0.19 \\
†WHR & $0.87(0.81-0.92)$ & $0.88(0.86-90)$ & 0.92 \\
\hline *Body mass index, "Waist hip ratio, BMl: Body mass index, WHR: Waist-hip ratio
\end{tabular}

the dynamic balance condition was easily available, easy to clean, easy to transport and easy to use for a child with DS when comparing with other balance measuring instruments such as balance master. Therefore, it is extremely useful in a school or community setting.

This is the first study that estimated timed measurements of balance in children with DS. Other studies that investigated standing balance in children and adults with DS did not report the maximum time required to maintain balance. ${ }^{[7,8,24]}$ Comparison of the timed static standing balance under eyes open and closed conditions reveals significant differences between typical children and children with DS. Typical children aged 4-9 years of age scored a maximum mean of $25.3 \mathrm{~s}$ under eyes open static and $6.3 \mathrm{~s}$ under eyes closed static conditions, whereas, in our study children with DS scored 4.21 and $2.68 \mathrm{~s}$ under eyes open static and eyes closed static, respectively. ${ }^{[25]}$ Similarly, large differences exist between healthy adults and those with disabilities as shown by the available evidence. A maximum mean of $30 \mathrm{~s}$ was reported in eyes closed static condition for healthy adults in comparison to adults with stroke who scored $2.8 \mathrm{~s}$ on the paretic and $6.6 \mathrm{~s}$ on the nonparetic leg during unilateral stance. ${ }^{[15,26]}$

The timed standing balance test has been tested for its psychometric properties in many neurologically disabled populations. The excellent test-retest reliability of eyes open static test was reported with an ICC $=0.87-0.98$ among individuals with congenital myotonic dystrophy type 1 aged $20-60$ years. ${ }^{[16]}$ However, the timed balance test during unilateral stance was not reliable for adults with stroke. ${ }^{[15]}$ In our study, both SDSBT had excellent absolute and relative reliability as inferred from the high ICC and low SEM values. The differences in the reliability estimates of the timed standing balance test can be attributed to the differences in age and health conditions. Moreover, some studies used arithmetic mean while the other used geometric mean to report the results, which might have misrepresented the actual differences between studies.

One of the major strength of this study was to pay utmost attention to the methodological considerations. First, we

Table 2: Geometric mean, median, absolute reliability, relative reliability, and sensitivity to change of the timed standing balance test

\begin{tabular}{lcccccc}
\hline Balance test & Geometric mean (95\% Cl) (sec) & Median (range) (sec) & ICC & SEM (sec) & 95\% SEM (sec) & SRC (sec) \\
\hline EOS & $4.21(2.08-5.63)$ & $6.4(1.2-28.9)$ & 0.93 & 0.46 & 0.90 & 1.27 \\
ECS & $2.68(1.59-4.57)$ & $2.15(1.3-9.4)$ & 0.91 & 0.59 & 1.15 \\
EOD & $2.67(1.55-5.33)$ & $2.25(1.2-10.7)$ & 0.92 & 0.21 & 0.41 \\
ECD & $1.57(1.05-3.09)$ & $1.6(0.7-4.6)$ & 0.91 & 0.22 & 0.43 \\
\hline
\end{tabular}

EOS: Eyes open static, ECS: Eyes closed static, EOD: Eyes open dynamic, ECD: Eyes closed dynamic, Cl: Confidence interval, ICC: Intraclass correlation coefficient, SEM: Standard error of measurement, SRC: Smallest real change 
randomized the order of executing four test conditions to control the effect of the ascending order of difficulty. Second, the reliability was calculated both in terms of ICC and SEM that made the interpretation of results more meaningful.

\section{Study limitations}

First, a sample size of nine children might not be a representative of children with DS. Second, children with DS were recruited from a single school that might influence the generalizability of results. Third, the assessor was not blinded to the second and third assessment. Nevertheless, this study was well-designed and adds evidence to the current literature that timed standing balance test is sensitive to change and can be used to clinically assess standing balance in children with DS. As no comparison was used, we cannot advocate the use of timed standing balance test over other measures. Further research may investigate the reliability and validity of the timed standing balance test in younger children or adults with DS. Concurrent validity of the timed standing test in comparison with other measures may also add to its utility in this population.

\section{Conclusion}

Timed static and dynamic balance test may be an appropriate clinical measure to be used in children with DS. Absolute and relative reliability appears to be adequate for all four conditions - eyes open static, eyes closed static, eyes open dynamic, and eyes closed dynamic. Based on the SRC values, it can be suggested that this test is sensitive to change under all static and dynamic balance conditions.

\section{Declaration of patient consent}

The authors certify that they have obtained all appropriate patient consent forms. In the form the patient(s) has/have given his/her/their consent for his/her/their images and other clinical information to be reported in the journal. The patients understand that their names and initials will not be published and due efforts will be made to conceal their identity, but anonymity cannot be guaranteed.

\section{Acknowledgments}

The authors are very much thankful to the children, their parents, and the head of Chethana Special School, Karkala, India, who gave their valuable time and cooperation. Special thanks to Ms. Viveta Priya Aranha, BBM, MBA, Karkala, India for her statistical assistance. This study was presented at the Synery-2014, National Physiotherapy Convention, Organised by Mohamed Sathak AJ College of Physiotherapy, Chennai, India on $27^{\text {th }}$ September, 2014.

\section{Financial support and sponsorship}

The foam pad used in this study is sponsored by Mrs. Gracy Lobo and Mr. Lancy Lobo, Almana General Hospital, Saudi Arabia and the transportation to the identified school by Mr. Valerian Clement Aranha, Vencita Villa, Nakre, Karkala, India.

\section{Conflicts of interest}

There are no conflicts of interest.

\section{References}

1. Presson AP, Partyka G, Jensen KM, Devine OJ, Rasmussen SA, McCabe LL, et al. Current estimate of Down syndrome population prevalence in the United States. J Pediatr 2013;163:1163-8.

2. Carvalho RL, Vasconcelos DA. Motor behavior in Down syndrome: A typical sensoriomotor control. In: Dey S, editor. Prenatal Diagnosis and Screening for Down Syndrome. Rijeka, Croatia: Intech; 2001. p. 33-9.

3. Shields N, Taylor NF, Fernhall B. A study protocol of a randomised controlled trial to investigate if a community based strength training programme improves work task performance in young adults with Down syndrome. BMC Pediatr 2010;10:17

4. Oates A, Bebbington A, Bourke J, Girdler S, Leonard H. Leisure participation for school-aged children with Down syndrome. Disabil Rehabil 2011;33:1880-9.

5. Shields N, Taylor NF, Wee E, Wollersheim D, O'Shea SD, Fernhall B. A community-based strength training programme increases muscle strength and physical activity in young people with Down syndrome: A randomised controlled trial. Res Dev Disabil 2013;34:4385-94.

6. Shields N, Taylor NF, Dodd KJ. Effects of a community-based progressive resistance training program on muscle performance and physical function in adults with Down syndrome: A randomized controlled trial. Arch Phys Med Rehabil 2008;89:1215-20.

7. Villarroya MA, González-Agüero A, Moros-García T, de la Flor Marín M, Moreno LA, Casajús JA. Static standing balance in adolescents with Down syndrome. Res Dev Disabil 2012;33:1294-300.

8. Villamonte R, Vehrs PR, Feland JB, Johnson AW, Seeley MK, Eggett D. Reliability of 16 balance tests in individuals with Down syndrome. Percept Mot Skills 2010;111:530-42.

9. Nolan L, Grigorenko A, Thorstensson A. Balance control: Sex and age differences in 9- to 16-year-olds. Dev Med Child Neurol 2005;47:449-54.

10. Wolff DR, Rose J, Jones VK, Bloch DA, Oehlert JW, Gamble JG. Postural balance measurements for children and adolescents. J Orthop Res 1998;16:271-5.

11. Bruton A, Conway JH, Holgate ST. Reliability: What is it, and how is it measured? Physiotherapy 2000;86:94-9.

12. Atkinson G, Nevill AM. Statistical methods for assessing measurement error (reliability) in variables relevant to sports medicine. Sports Med 1998;26:217-38.

13. Horak FB. Clinical measurement of postural control in adults. Phys Ther 1987;67:1881-5

14. Emery CA, Cassidy JD, Klassen TP, Rosychuk RJ, Rowe BB. Development of a clinical static and dynamic standing balance measurement tool appropriate for use in adolescents. Phys Ther 2005;85:502-14.

15. Bohannon RW, Walsh S, Joseph MC. Ordinal and timed balance measurements: Reliability and validity in patients with stroke. Clin Rehabil 1993;7:9-13.

16. Hammarén E, Ohlsson JA, Lindberg C, Kjellby-Wendt G. Reliability of static and dynamic balance tests in subjects with myotonic dystrophy type 1. Adv Physiother 2012;14:48-54.

17. Shieh G. Sample size requirements for the design of reliability studies: Precision consideration. Behav Res Methods 2014;46:808-22.

18. Donner A, Eliasziw M. Sample size requirements for reliability studies. Stat Med 1987;6:441-8. 
19. Altman DG. Practical Statistics for Medical Research. London, UK: Chapman and Hall; 1991.

20. Shrout PE, Fleiss JL. Intraclass correlations: Uses in assessing rater reliability. Psychol Bull 1979;86:420-8.

21. Stratford PW. Getting more from the literature: Estimating the standard error of measurement from reliability studies. Physiother Can 2004;56:27-30.

22. Beckerman H, Roebroeck ME, Lankhorst GJ, Becher JG, Bezemer PD, Verbeek AL. Smallest real difference, a link between reproducibility and responsiveness. Qual Life Res 2001;10:571-8.

23. Van Baalen B, Odding E, van Woensel MP, Roebroeck ME. Reliability and sensitivity to change of measurement instruments used in a traumatic brain injury population. Clin Rehabil 2006;20:686-700.

24. Webber A, Virji-Babul N, Edwards R, Lesperance M. Stiffness and postural stability in adults with Down syndrome. Exp Brain Res 2004;155:450-8.

25. Atwater SW, Crowe TK, Deitz JC, Richardson PK. Interrater and test-retest reliability of two pediatric balance tests. Phys Ther 1990;70:79-87.

26. Bohannon RW, Larkin PA, Cook AC, Gear J, Singer J. Decrease in timed balance test scores with aging. Phys Ther 1984;64:1067-70. 